(C) The Authors 2014. This is an Open Access article, distributed under the terms of the Creative Commons Attribution licence (http:// creativecommons.org/licenses/by/3.0/), which permits unrestricted re-use, distribution, and reproduction in any medium, provided the original work is properly cited.

\title{
Dietary effects on human gut microbiome diversity
}

\author{
Zhenjiang $\mathrm{Xu}^{1}$ and Rob Knight ${ }^{1,2 *}$ \\ ${ }^{1}$ Biofrontiers Institute, University of Colorado, Boulder, CO, USA \\ ${ }^{2}$ Howard Hughes Medical Institute, University of Colorado, Boulder, CO, USA \\ (Submitted 22 November 2013 - Final revision received 28 October 2014 - Accepted 17 November 2014 - First published online 11 December 2014)
}

\section{Abstract}

The human gut harbours diverse and abundant microbes, forming a complex ecological system that interacts with host and environmental factors. In this article, we summarise recent advances in microbiome studies across both Western and non-Western populations, either in cross-sectional or longitudinal surveys, and over various age groups, revealing a considerable diversity and variability in the human gut microbiome. Of all the exogenous factors affecting gut microbiome, a long-term diet appears to have the largest effect to date. Recent research on the effects of dietary interventions has shown that the gut microbiome can change dramatically with diet; however, the gut microbiome is generally resilient, and short-term dietary intervention is not typically successful in treating obesity and malnutrition. Understanding the dynamics of the gut microbiome under different conditions will help us diagnose and treat many diseases that are now known to be associated with microbial communities.

\section{Key words: Microbiome: Diet: Gut}

It is estimated that human body contains as many as $10^{14}$ microbial cells ${ }^{(1)}$, and our appreciation of their contribution to host physiology, disease and behaviour is increasing rapidly ${ }^{(2-5)}$. This complex community, collectively known as the microbiota (their genes are known as the microbiome), contains diverse viruses, bacteria, archaea and eukaryotes ${ }^{(3,6)}$. Recent advances in high-throughput sequencing technology, together with the development of bioinformatics techniques, have sparked a tremendous explosion of culture-independent microbiome studies (i.e. studies that do not rely on culturebased techniques, which usually capture only a small fraction of microbial diversity) providing a profound insight into the role of the microbiota in human health ${ }^{(7,8)}$. For example, gut microbes train the immune system $^{(9)}$, protect against opportunistic pathogens ${ }^{(10)}$, harvest nutrients and energy from diet $^{(11)}$, and ferment non-digestible carbohydrates ${ }^{(12-14)}$. The disruption of the normal gut microbiota (dysbiosis) is associated with obesity ${ }^{(15,16)}$, diabetes ${ }^{(17)}$, various inflammatory bowel diseases $(\mathrm{IBD})^{(18,19)}$ and autoimmune diseases ${ }^{(20,21)}$. We are beginning to understand the baseline states for a healthy microbiota (or, rather, for the diverse array of healthy microbiota found in different healthy people), and in contrast, what constitutes a bad microbiota, by studying the taxonomy of the constituent organisms (revealing who is there) and their genes (revealing what they are capable of doing). Many factors, either exogenous or endogenous, affect the composition of the gut microbiota. These factors include host genotype ${ }^{(22)}$, age $^{(2)}$ and sex ${ }^{(23)}$. However, of all the environmental factors studied to date, diet has the largest known impact on the gut microbiota. Revealing the complex interactions between these factors and microbiota may ultimately help us modulate our microbiome to diagnose and treat microbiome-associated diseases in a personalised way, restoring a healthy microbial community.

\section{Variability of the human gut microbiome across populations and over time}

The human gut microbiota is seeded during birth and mainly develops over the first 3 years of life ${ }^{(24)}$. From birth, neonates are exposed to microbes from a variety of sources, and the initial colonisation of their guts depends on the microbes first encountered. The initial composition of the gut microbiota depends on the mode of delivery: babies delivered vaginally harbour gut microbiota resembling microbial communities found in their mothers' vaginas, whereas those

Abbreviations: IBD, inflammatory bowel diseases; MetaHIT, Metagenomics of the Human Intestinal Tract.

* Corresponding author: R. Knight, email rob.knight@colorado.edu

This article was published as part of the WALTHAM International Nutritional Sciences Symposium Proceedings 2013.

This paper was published as part of a supplement to British Journal of Nutrition, publication of which was supported by an unrestricted educational grant from Mars Incorporated. The papers included in this supplement were invited by the Guest Editor and have undergone the standard journal formal review process. They may be cited. 
born via Cesarean section apparently acquire microbes from the skin, dominated by taxa such as Propionibacterium and Staphylococcus ${ }^{(25)}$. The feeding mode also influences the infant gut microbiota. Breast milk contains nutrients, maternal antibodies and also diverse commensal maternal bacteria including bifidobacteria and lactobacilli ${ }^{(26)}$. Compared with formula-fed infants, breast-fed infants have lower levels of Atopobium and higher levels of Bifidobacterium ${ }^{(27)}$. The diversity in the baby's gut is initially low and increases during development. Over the first 3 years of life, the composition of the microbial community becomes more adult-like, and major microbial shifts are associated with key events such as the introduction of solid food ${ }^{(24)}$.

Although the adult gut microbiota are relatively stable within a person (in that serial samples from the same person typically resemble each other more closely than do samples from different people) and are typically dominated by members of the Bacteroidetes, Firmicutes and Actinobacteria, they show great interpersonal and intrapersonal variability. For example, fewer than $50 \%$ of bacterial taxa are shared between monozygotic twins at the species level ${ }^{(28)}$. We are starting to appreciate this variability to a greater extent because of the numerous studies facilitated by the methods developed in large-scale projects such as the Human Microbiome Project ${ }^{(7,8)}$ and the Metagenomics of the Human Intestinal Tract (MetaHIT) ${ }^{(29)}$. It has been argued that the gut microbiota can be classified into three broad clusters, or 'enterotypes', based on the dominant presence of Bacteroides, Prevotella or Ruminococcus genera $^{(30)}$, although statistical support for the three-cluster model is weak and has generally not been replicated in later studies. The idea is controversial, especially because the model of discrete clusters has a clear alternative in terms of continuous gradients in the major taxa that are well supported $^{(31-34)}$. Intriguingly, despite the considerable variability in the composition of the gut microbiota, metagenomic shotgun sequencing demonstrates that these diverse communities share a core set of gene functions in the microbiome ${ }^{(35)}$.

The gut microbial community changes with age. In crosssectional studies, we see an apparently smooth change in the gut microbiota in the first 3 years of life, followed by relatively subtle changes thereafter. Although few studies have specifically examined the gut microbiota in old age, recent studies on elderly population confirm that they harbour distinct gut microbial communities from younger adults ${ }^{(36)}$, and the composition of the microbiota in the elderly subjects correlates significantly with measures of frailty, co-morbidity and nutrition ${ }^{(37)}$. Lachnospiraceae and microbial genes involved in the production of SCFA were enriched in elderly subjects living in the community compared with those in long-term care. Another study performing functional profiling of the gut microbiota found an increase in proteolytic function and a decrease in saccharolytic function, in addition to the loss of genes for SCFA production associated with ageing ${ }^{(38)}$ However, more studies, and especially prospective longitudinal studies, are needed to clarify how diet and the gut microbiota affect the ageing process and how the gut microbiota are in turn affected by aging itself.
Understanding the variability of the microbiota between people and over time is a prerequisite to discriminating disease states from normal perturbations. Caporaso et al. ${ }^{(39)}$ densely sampled microbiota from three body sites (tongue, gut and skin) of two healthy subjects for 6 and 15 months. This time series shows the remarkable temporal dynamics of the gut microbiota of individuals. Although the difference between gut samples from an individual is smaller than those from different individuals or different body sites, there seems to be no core set of taxa present at all time points. However, Faith et $a l{ }^{(40)}$ found that in faecal samples sequenced much more deeply, on an average $60 \%$ of strains in the gut can persist over a long period up to 5 years when only a few time points are evaluated, suggesting that either the dynamic range of abundance of particular taxa is large (requiring very deep sequencing to recover them) or strains can be lost but recolonise the host. This temporal consistency assumes that other factors that could affect the microbiota, such as diet and disease status, remain relatively constant.

Most studies of the gut microbiota have been focused on Western populations in Europe, the USA and Canada. It is important to expand these studies to non-Western diet populations in order to fully understand the range of variation of the gut microbiota and how gut microbes have co-evolved with humans. For example, the Japanese population has a unique gene coding for the enzyme porphyranase in the gut bacterium Bacteroides plebeius, potentially transferred from marine Bacteroides spp. that naturally degrade seaweed, to facilitate the digestion of this component of the Japanese, but not, historically, the US diet ${ }^{(41)}$. Gut microbiota of children in a rural African village in Burkina Faso showed an increase in Bacteroidetes and a decrease in Firmicutes relative to European children ${ }^{(12)}$. This pattern was also found in a broader cross-cultural comparison of the gut microbiota of people living in the Venezuelan Amazon, rural Malawi and the USA, suggesting that carbohydrate-rich diets increase the amount of Prevotella ${ }^{(2)}$. The Hadza hunter-gatherers in Tanzania also harbour unique compositions of microbiota, presumably as a result of their foraging lifestyle ${ }^{(42)}$. All these studies demonstrate the heterogeneity of gut microbiota across geographically and culturally diverse populations, although to date not enough populations have been studied to resolve which of these differences are due to diet, which to host genetics and which to environmental exposures, all of which differ among these populations.

\section{Dietary effects on the gut microbiome}

The quantitative contributions of host genetics, environmental factors and diet to shaping the gut microbiota remain largely unknown. Significant associations between host genotypes and their gut microbiota composition have been reported in both human and mouse studies (see Spor et al. ${ }^{(43)}$ for a comprehensive recent review). The environment also plays an important role in establishing and modifying the gut microbiota. For example, the close physical contact between humans and companion animals facilitates the acquisition and exchange of microbes. A study of skin, oral and gut microbiomes 
showed that the microbial communities of cohabiting family members resemble each other and that adults share more microbial taxa with their own dogs than they do with others' dogs ${ }^{(44)}$. Having a dog, and the resulting exposure to a diverse microbial community, can be beneficial. Pet ownership in the early, but not later, years of life ${ }^{(45)}$ is associated with a significant decrease in the risk of allergic disease ${ }^{(46)}$.

As discussed earlier, dietary effects on the gut microbiota and health are often confounded by variation in host genotypes and environmental exposures. Nevertheless, accumulating evidence has suggested that long-term diet is a primary driver of the gut microbiota. As an extreme example, two co-evolution studies of mammals and their gut microbiota has found that both gut microbiota composition and functions are adapted to their diet (herbivorous, carnivorous and omnivorous) ${ }^{(47,48)}$. In another more recent study, the convergently evolved composition of the gut microbiome in ant-eating mammals, despite their phylogenetic diversity, strongly suggests that diet is the major force shaping their microbiota ${ }^{(49)}$. Wu et al. ${ }^{(31)}$ showed that the overall structure of the human gut microbiota, at least in a Western population, seems to be driven mainly by long-term dietary effects: Prevotella was enriched in people with high-fibre and high-carbohydrate diets, whereas Bacteroides was associated with a typical 'Western' diet high in protein and fat. The studies highlighted in the previous section also suggest that differences between the gut microbial communities of rural populations and Westerners are largely linked to differences in diet $^{(2,12,42)}$.

Fortunately, we can examine dietary effects more directly with techniques that constrain other interacting factors. Studies of the faecal microbiomes of human monozygotic and dizygotic twins have been helpful in identifying the effects of diet on gut microbiota controlling for host genetics ${ }^{(50)}$. In addition, gnotobiotic mice (initially germ-free mice colonised with either a defined set of microbial strains or with a donor faecal sample) provides a well-defined, powerful animal model of the human gut ecosystem to elucidate the effects of nutrients on the microbiome, including taxonomic composition, microbial and host gene expression, and, ultimately, host physiological status in response to controlled changes in diet ${ }^{(15,51)}$. Using the gnotobiotic mouse model, a recent study found that obese or lean phenotypes can be transmitted to germ-free mice by inoculating them with the gut microbiota from human twins discordant for obesity and that the obese phenotype of the mouse transplanted with the obese microbiota can be rescued by the invasion of a particular species of Bacteroidetes from the mouse transplanted with lean microbiota when they are co-housed $^{(14)}$. This rescue and invasion is diminished when the mice are fed with a diet high in saturated fats and low in vegetables and fruit, confirming the dependency of the gut microbiota on diet.

Arguably, diet is among the most easily controlled factors that can potentially manipulate the gut microbiota. Studying the resilience of the microbiota and its patterns of change, during and after dietary intervention, could allow the design of effective nutrition therapy. Ley et $a l .{ }^{(52)}$ have shown that the ratio of Bacteroidetes:Firmicutes increases in the gut microbiota of people consuming either a fat-restricted or a carbohydrate-restricted low-energy diet for a year and that people who lost more weight showed larger changes in the ratios of these taxa. Two other short-term controlled feeding experiments found that the changes in microbiota composition can be rapid (within $24 \mathrm{~h}$ ), reproducible and can overwhelm interpersonal variability with a sufficiently extreme diet ${ }^{(31,53)}$. A dietary intervention study of obese or overweight individuals found that the gene richness (the number of gut microbial genes) is increased and the metabolic status is improved in individuals with low gut microbial gene richness, after a 6-week low-energy, highprotein diet followed by another 6-week weight maintenance diet $^{(54)}$, but this improvement is very limited for individuals with already high gut microbial gene richness. Dietary interventions in malnourished humans are not always successful, and the variation may be explained by individual differences in the gut microbiota. Studies of Malawian and Bangladeshi subjects with severe acute malnutrition have shown that their gut microbiota are resilient and often regress back to the state before the food intervention ${ }^{(50,55)}$, suggesting that longer intervention or a repair of the gut microbiota is needed to effectively treat malnutrition in these cases.

\section{Conclusions}

It remains a challenge to identify the key pathogenic microbiota and to establish a causal (rather than associative) relationship between specific microbes or community states and a given physiological or disease phenotype ${ }^{(5)}$. Exactly what role does the microbiota play in obesity, diabetes and other diseases? Is the microbiota shift a result of changes in gut environment or a cause? Does microbiota initiate the effect, or mediate it? Can we design therapeutic food interventions or faecal transplantations to restore a healthy microbiota and cure diseases that stem from dysbiosis? These questions are largely unanswered and will require more mechanistic studies of the gut microbiota. Encouragingly, a recent randomised clinical trial of faecal transplantation demonstrated that it is much more effective at curing recurring infections of Clostridium difficile $^{(56)}$ than traditional antibiotic treatment.

Vast resources have been invested in studies of mouse and human microbiota such as the Human Microbiome Project $^{(7,57,58)}$ and the MetaHIT project ${ }^{(29,59)}$. These projects and their associated technologies have led to a greater understanding of the function of the microbiome. Many of the tools developed and the conclusions reached for the human microbiome can be readily transferred to other host-associated studies. Similar to the human gut microbiota, Firmicutes and Bacteroidetes are also the predominant taxa in horses ${ }^{(60)}$, guinea pig ${ }^{(61)}$, cats and dogs ${ }^{(62)}$. Gastrointestinal microbiome dysbiosis is common in canine and feline diseases, such as diarrhoea and IBD, and the alterations in the bacterial communities of dogs and cats with IBD resemble, at least partially, the dysbiosis in human with chronic intestine inflammation $^{(63)}$. For example, Clostridium clusters XIVa and IV, which produce beneficial SCFA, are depleted in both humans and dogs with IBD $^{(64)}$. 
The interactions of host genetics, diet and the gut microbiota are thus convoluted and multi-faceted. In many cases, the effect size may be small compared with the huge variability intrinsic in the data. It will require a large number of samples to reveal such small effects. Citizen science projects such as the American Gut Project (http://www.microbio.me/ americangut/) launched in 2013 provide opportunities for the general public to participate. The American Gut Project is one of the largest crowd-funded citizen science projects, with over 3600 participants and 3800 samples collected to date. Such large-scale studies with greater statistical power will allow us to examine the subtle contributions of diet and other environmental factors on the gut microbiota from an integrated perspective.

\section{Acknowledgements}

This work is supported in part by the Howard Hughes Medical Institute and the National Institutes of Health.

The authors declare no competing financial interests.

The authors' contributions are as follows: Z. X. wrote the manuscript; both authors conceived and edited the manuscript.

\section{References}

1. Whitman WB, Coleman DC \& Wiebe WJ (1998) Prokaryotes: the unseen majority. Proc Natl Acad Sci 95, 6578-6583.

2. Yatsunenko T, Rey FE, Manary MJ, et al. (2012) Human gut microbiome viewed across age and geography. Nature 486, 222-227.

3. Lozupone CA, Stombaugh JI, Gordon JI, et al. (2012) Diversity, stability and resilience of the human gut microbiota. Nature 489, 220-230.

4. Clemente JC, Ursell LK, Parfrey LW, et al. (2012) The impact of the gut microbiota on human health: an integrative view. Cell 148, 1258-1270.

5. Zhao L (2013) The gut microbiota and obesity: from correlation to causality. Nat Rev Microbiol 11, 639-647.

6. Eckburg PB, Bik EM, Bernstein CN, et al. (2005) Diversity of the human intestinal microbial flora. Science 308, 1635-1638.

7. Turnbaugh PJ, Ley RE, Hamady M, et al. (2007) The Human Microbiome Project. Nature 449, 804-810.

8. Human Microbiome Project Consortium (2012) A framework for human microbiome research. Nature 486, 215-221.

9. Chow J, Lee SM, Shen Y, et al. (2010) Host-bacterial symbiosis in health and disease. Adv Immunol 107, 243-274.

10. Fukuda S, Toh H, Hase K, et al. (2011) Bifidobacteria can protect from enteropathogenic infection through production of acetate. Nature 469, 543-547.

11. Sonnenburg JL, Xu J, Leip DD, et al. (2005) Glycan foraging in vivo by an intestine-adapted bacterial symbiont. Science 307, 1955-1959.

12. De Filippo C, Cavalieri D, Paola MD, et al. (2010) Impact of diet in shaping gut microbiota revealed by a comparative study in children from Europe and rural Africa. Proc Natl Acad Sci U S A 107, 14691-14696.

13. Cantarel BL, Lombard V \& Henrissat B (2012) Complex carbohydrate utilization by the healthy human microbiome. PLOS ONE 7, e28742.
14. Ridaura VK, Faith JJ, Rey FE, et al. (2013) Gut microbiota from twins discordant for obesity modulate metabolism in mice. Science 341, 1241214.

15. Turnbaugh PJ, Ley RE, Mahowald MA, et al. (2006) An obesity-associated gut microbiome with increased capacity for energy harvest. Nature 444, 1027-1031.

16. Kallus SJ \& Brandt LJ (2012) The intestinal microbiota and obesity. J Clin Gastroenterol 46, 16-24.

17. Qin J, Li Y, Cai Z, et al. (2012) A metagenome-wide association study of gut microbiota in type 2 diabetes. Nature 490, 55-60

18. Gevers D, Kugathasan S, Denson LA, et al. (2014) The treatment-naive microbiome in new-onset Crohn's disease. Cell Host Microbe 15, 382-392.

19. Morgan XC, Tickle TL, Sokol H, et al. (2012) Dysfunction of the intestinal microbiome in inflammatory bowel disease and treatment. Genome Biol 13, R79.

20. Giongo A, Gano KA, Crabb DB, et al. (2011) Toward defining the autoimmune microbiome for type 1 diabetes. ISME J 5, 82-91.

21. Proal AD, Albert PJ \& Marshall TG (2013) The human microbiome and autoimmunity. Curr Opin Rheumatol 25, 234-240.

22. Benson AK, Kelly SA, Legge R, et al. (2010) Individuality in gut microbiota composition is a complex polygenic trait shaped by multiple environmental and host genetic factors. Proc Natl Acad Sci 107, 18933-18938.

23. Bolnick DI, Snowberg LK, Hirsch PE, et al. (2014) Individual diet has sex-dependent effects on vertebrate gut microbiota. Nat Commun 5, 4500.

24. Koenig JE, Spor A, Scalfone N, et al. (2011) Succession of microbial consortia in the developing infant gut microbiome. Proc Natl Acad Sci 108, 4578-4585.

25. Dominguez-Bello MG, Costello EK, Contreras M, et al. (2010) Delivery mode shapes the acquisition and structure of the initial microbiota across multiple body habitats in newborns. Proc Natl Acad Sci U S A 107, 11971-11975.

26. Jost T, Lacroix C, Braegger C, et al. (2013) Assessment of bacterial diversity in breast milk using culture-dependent and culture-independent approaches. Br J Nutr 110, $1253-1262$.

27. Bezirtzoglou E, Tsiotsias A \& Welling GW (2011) Microbiota profile in feces of breast- and formula-fed newborns by using fluorescence in situ hybridization (FISH). Anaerobe 17, 478-482.

28. Turnbaugh PJ, Quince C, Faith JJ, et al. (2010) Organismal, genetic, and transcriptional variation in the deeply sequenced gut microbiomes of identical twins. Proc Natl Acad Sci 107, 7503-7508.

29. Ehrlich SD (2011) MetaHIT: The European Union Project on metagenomics of the human intestinal tract. In Metagenomics of the Human Body, pp. 307-316 [KE Nelson, editor]. New York, NY: Springer.

30. Arumugam M, Raes J, Pelletier E, et al. (2011) Enterotypes of the human gut microbiome. Nature 473, 174-180.

31. Wu GD, Chen J, Hoffmann C, et al. (2011) Linking long-term dietary patterns with gut microbial enterotypes. Science 334, 105-108.

32. Knights D, Ward TL, McKinlay CE, et al. (2014) Rethinking 'Enterotypes'. Cell Host Microbe 16, 433-437.

33. Jeffery IB, Claesson MJ, O'Toole PW, et al. (2012) Categorization of the gut microbiota: enterotypes or gradients? Nat Rev Microbiol 10, 591-592.

34. Koren O, Knights D, Gonzalez A, et al. (2013) A guide to enterotypes across the human body: meta-analysis of 
microbial community structures in human microbiome datasets. PLOS Comput Biol 9, e1002863

35. Turnbaugh PJ, Hamady M, Yatsunenko T, et al. (2008) A core gut microbiome in obese and lean twins. Nature 457, 480-484.

36. Claesson MJ, Cusack S, O'Sullivan O, et al. (2011) Composition, variability, and temporal stability of the intestinal microbiota of the elderly. Proc Natl Acad Sci 108, 4586-4591.

37. Claesson MJ, Jeffery IB, Conde S, et al. (2012) Gut microbiota composition correlates with diet and health in the elderly. Nature 488, 178-184.

38. Rampelli S, Candela M, Turroni S, et al. (2013) Functional metagenomic profiling of intestinal microbiome in extreme ageing. Aging 5, 902-912.

39. Caporaso JG, Lauber CL, Costello EK, et al. (2011) Moving pictures of the human microbiome. Genome Biol 12, R50.

40. Faith JJ, Guruge JL, Charbonneau M, et al. (2013) The longterm stability of the human gut microbiota. Science 341, 1237439.

41. Hehemann J-H, Correc G, Barbeyron T, et al. (2010) Transfer of carbohydrate-active enzymes from marine bacteria to Japanese gut microbiota. Nature 464, 908-912.

42. Schnorr SL, Candela M, Rampelli S, et al. (2014) Gut microbiome of the Hadza hunter-gatherers. Nat Commun 5, 3654.

43. Spor A, Koren O \& Ley R (2011) Unravelling the effects of the environment and host genotype on the gut microbiome. Nat Rev Microbiol 9, 279-290.

44. Song SJ, Lauber C, Costello EK, et al. (2013) Cohabiting family members share microbiota with one another and with their dogs. elife 2, e00458.

45. Wegienka G, Johnson CC, Havstad S, et al. (2011) Lifetime dog and cat exposure and dog- and cat-specific sensitization at age 18 years. Clin Exp Allergy 41, 979-986.

46. Fujimura KE, Johnson CC, Ownby DR, et al. (2010) Man's best friend? The effect of pet ownership on house dust microbial communities. J Allergy Clin Immunol 126, 410-412, 412.e1-3.

47. Ley RE, Hamady M, Lozupone C, et al. (2008) Evolution of mammals and their gut microbes. Science 320, 1647-1651.

48. Muegge BD, Kuczynski J, Knights D, et al. (2011) Diet drives convergence in gut microbiome functions across mammalian phylogeny and within humans. Science 332, 970-974.

49. Delsuc F, Metcalf JL, Wegener Parfrey L, et al. (2013) Convergence of gut microbiomes in myrmecophagous mammals. Mol Ecol 23, 1301-1317.
50. Smith MI, Yatsunenko T, Manary MJ, et al. (2013) Gut microbiomes of Malawian twin pairs discordant for kwashiorkor. Science 339, 548-554.

51. Turnbaugh PJ, Ridaura VK, Faith JJ, et al. (2009) The effect of diet on the human gut microbiome: a metagenomic analysis in humanized gnotobiotic mice. Sci Transl Med 1, Gra14.

52. Ley RE, Turnbaugh PJ, Klein S, et al. (2006) Microbial ecology: human gut microbes associated with obesity. Nature 444, 1022-1023.

53. David LA, Maurice CF, Carmody RN, et al. (2014) Diet rapidly and reproducibly alters the human gut microbiome. Nature $\mathbf{5 0 5}, 559-563$.

54. Cotillard A, Kennedy SP, Kong LC, et al. (2013) Dietary intervention impact on gut microbial gene richness. Nature $\mathbf{5 0 0}$, $585-588$

55. Subramanian S, Huq S, Yatsunenko T, et al. (2014) Persistent gut microbiota immaturity in malnourished Bangladeshi children. Nature 510, 417-421.

56. Van Nood E, Vrieze A, Nieuwdorp M, et al. (2013) Duodenal infusion of donor feces for recurrent Clostridium difficile. N Engl J Med 368, 407-415.

57. Human Microbiome Project Consortium (2012) Structure, function and diversity of the healthy human microbiome. Nature 486, 207-214.

58. Proctor LM (2011) The Human Microbiome Project in 2011 and beyond. Cell Host Microbe 10, 287-291.

59. Qin J, Li R, Raes J, et al. (2010) A human gut microbial gene catalogue established by metagenomic sequencing. Nature 464, 59-65.

60. Costa MC, Arroyo LG, Allen-Vercoe E, et al. (2012) Comparison of the fecal microbiota of healthy horses and horses with colitis by high throughput sequencing of the V3-V5 region of the 16S rRNA gene. PLOS ONE 7, e41484.

61. Hildebrand F, Ebersbach T, Nielsen HB, et al. (2012) A comparative analysis of the intestinal metagenomes present in guinea pigs (Cavia porcellus) and humans (Homo sapiens). BMC Genomics 13, 514.

62. Handl S, Dowd SE, Garcia-Mazcorro JF, et al. (2011) Massive parallel 16S rRNA gene pyrosequencing reveals highly diverse fecal bacterial and fungal communities in healthy dogs and cats. FEMS Microbiol Ecol 76, 301-310.

63. Suchodolski JS, Dowd SE, Wilke V, et al. (2012) 16S rRNA gene pyrosequencing reveals bacterial dysbiosis in the duodenum of dogs with idiopathic inflammatory bowel disease. PLOS ONE 7, e39333.

64. Suchodolski JS, Markel ME, Garcia-Mazcorro JF, et al. (2012) The fecal microbiome in dogs with acute diarrhea and idiopathic inflammatory bowel disease. PLOS ONE 7, e51907. 